\title{
Alterstice
}

Revue internationale de la recherche interculturelle

International Journal of Intercultural Research

Revista International de la Investigacion Intercultural

\section{Déqualification des femmes migrantes en Suisse : mécanismes sous-jacents et effets psychologiques}

\section{Betty Goguikian Ratcliff, Claudio Bolzman et Théogène-Octave Gakuba}

Volume 4, numéro 2, 2014

Santé mentale et sociétés plurielles

URI : https://id.erudit.org/iderudit/1077426ar

DOI : https://doi.org/10.7202/1077426ar

Aller au sommaire du numéro

Éditeur(s)

Alterstice

ISSN

1923-919X (numérique)

Découvrir la revue

Citer cet article

Goguikian Ratcliff, B., Bolzman, C. \& Gakuba, T.-O. (2014). Déqualification des femmes migrantes en Suisse : mécanismes sous-jacents et effets psychologiques. Alterstice, 4(2), 63-76. https://doi.org/10.7202/1077426ar

\section{Résumé de l'article}

En Suisse et dans d'autres pays européens, la majorité des migrants légaux sont des femmes. Une proportion importante de ces dernières est en possession de diplômes d'un haut niveau de qualification, mais elles se trouvent souvent désavantagées sur le marché du travail du pays d'accueil. Cette revue de la littérature étudie le phénomène de la déqualification, c'est-à-dire un déclassement sur le marché de l'emploi des personnes par rapport à leur niveau de scolarité et à leurs expériences professionnelles précédentes. Ce phénomène est expliqué par les politiques migratoires du pays d'accueil d'une part, par les facteurs familiaux d'autre part, mais également par la discrimination. Diverses recherches ont montré que la femme se trouvant à la croisée de plusieurs motifs de discrimination, tels que le genre, le sexe et l'origine nationale (notamment celle des pays non membres de l'OCDE) est d'autant plus désavantagée lorsqu'il s'agit d'accéder à un emploi qualifié, et ceci d'une manière durable. La déqualification ainsi que la perception de la discrimination mènent souvent au sentiment d'autodépréciation et de dévalorisation et sont fortement associées à une mauvaise santé physique et à une détresse psychologique. d'utilisation que vous pouvez consulter en ligne.

https://apropos.erudit.org/fr/usagers/politique-dutilisation/ 


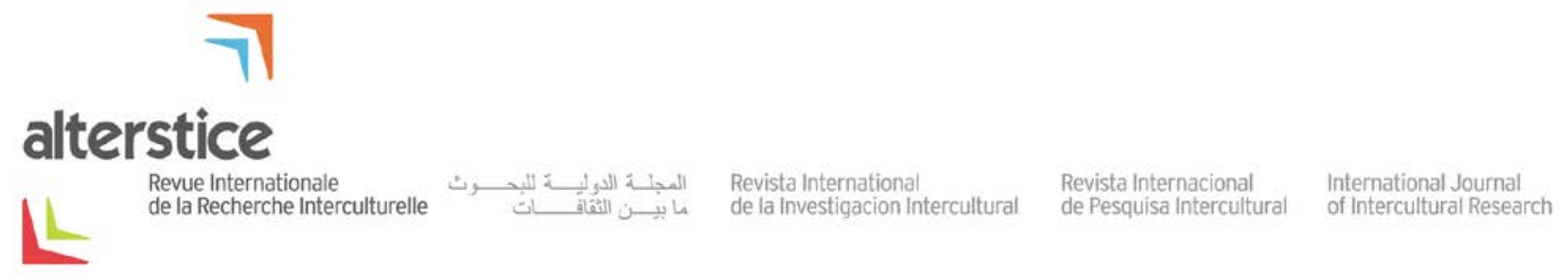

ARTICLE THÉMATIQUE

\title{
Déqualification des femmes migrantes en Suisse : mécanismes sous-jacents et effets psychologiques
}

\author{
Betty Goguikian Ratcliff ${ }^{1}$, Claudio Bolzman ${ }^{2}$ et Théogène-Octave Gakuba ${ }^{2}$
}

\section{Résumé}

En Suisse et dans d'autres pays européens, la majorité des migrants légaux sont des femmes. Une proportion importante de ces dernières est en possession de diplômes d'un haut niveau de qualification, mais elles se trouvent souvent désavantagées sur le marché du travail du pays d'accueil. Cette revue de la littérature étudie le phénomène de la déqualification, c'est-à-dire un déclassement sur le marché de l'emploi des personnes par rapport à leur niveau de scolarité et à leurs expériences professionnelles précédentes. Ce phénomène est expliqué par les politiques migratoires du pays d'accueil d'une part, par les facteurs familiaux d'autre part, mais également par la discrimination. Diverses recherches ont montré que la femme se trouvant à la croisée de plusieurs motifs de discrimination, tels que le genre, le sexe et l'origine nationale (notamment celle des pays non membres de l'OCDE) est d'autant plus désavantagée lorsqu'il s'agit d'accéder à un emploi qualifié, et ceci d'une manière durable. La déqualification ainsi que la perception de la discrimination mènent souvent au sentiment d'autodépréciation et de dévalorisation et sont fortement associées à une mauvaise santé physique et à une détresse psychologique.

\section{Rattachement des auteurs}

${ }^{1}$ Université de Genève, Suisse; ${ }^{2}$ Haute École Spécialisée de Suisse Occidentale (HES-SO Genève), Genève, Suisse.

\section{Correspondance}

betty.goguikian@unige.ch

\section{Mots clés}

femmes migrantes qualifiées; déqualification; marché du travail; santé mentale; discrimination perçue.

\section{Pour citer cet article}

Goguikian Ratcliff, B., Bolzman, C. et Gakuma, T.-O. (2014). Déqualification des femmes migrantes en Suisse: mécanismes sous-jacents et effets psychologiques. Alterstice, 4(2), 63-76. 


\section{Introduction}

Dans de nombreux pays du monde, la majorité des nouveaux arrivants légaux sont des femmes ${ }^{1}$. Dans la plupart des cas, l'immigration s'effectue pour des raisons de regroupement familial, l'obtention d'un droit de séjour se faisant sur la base de critères familiaux et conjugaux. De ce fait, même si une proportion importante de femmes migrantes est en possession de diplômes et d'un niveau de qualification élevé, elles restent "invisibles » et ne figurent pas dans les statistiques relatives au marché de l'emploi (Kofman, 2000).

Alors que le nombre d'entrées de femmes migrantes en Suisse et ailleurs en Europe dépasse souvent celui des hommes, les politiques migratoires ont tardé à mettre sur pied des programmes spécifiques destinés aux femmes, en particulier aux travailleuses migrantes. Les programmes existants destinés aux femmes migrantes ciblent en priorité les difficultés familiales et sociales rencontrées par celles qui se trouvent en situation de précarité, et concernent rarement la question de l'insertion professionnelle. Les stéréotypes de sexe, encore fortement ancrés, cantonnent la femme migrante à son rôle d'épouse et de mère, financièrement dépendante de son mari et gardienne des traditions, et ils la confinent à des domaines d'activité peu valorisants.

Or, la mobilité spatiale des femmes dans certains pays, y compris des femmes seules, est plus importante que celle des hommes (Amérique Latine, Philippines) (Bolzman, Carbajal et Mainardi, 2007). On omet souvent de souligner à quel point leur travail constitue un apport conséquent dans l'économie de leurs pays et participe aux échanges dans un monde globalisé (Chaïb, 2001). Un grand nombre de ces femmes sont qualifiées et connaissent, du moins temporairement, une situation de déqualification. Ce phénomène suscite actuellement de plus en plus d'intérêt et de préoccupation (Moreno-Fontes, 2008).

\section{Mondialisation, migration féminine et travail}

Depuis une quinzaine d'années, un courant de recherche en sociologie de la migration s'intéresse aux femmes en migration et au rapport entre genre et migration (Kofman, 2009; Morokvasic, 2003). L'un des premiers constats de ces travaux fut que les femmes représentent en quelque sorte la face cachée de la migration, seul l'homme étant reconnu comme actif. L'explication proposée renvoie à l'opposition bipolaire entre, d'une part, l'association de l'économique, de la sphère du travail (production sociale) et du masculin et, d'autre part, le socioculturel, la famille (reproduction sociale) et le féminin (Kofman, 2000 et 2004). Catarino et Morokvasic (2005) expliquent qu'afin de rendre les femmes socialement visibles, il a fallu procéder à un travail de déconstruction des représentations sociales et « poser le regard là où l'on ne s'attendait pas à les trouver : la sphère du travail » (p. 7).

Les secteurs d'activité traditionnellement investis par les femmes sont les premiers à avoir été étudiés : service domestique, emplois du care, prostitution. Ces activités peu qualifiées, considérées comme des extensions du rôle exercé par les migrantes dans la sphère privée, ont monopolisé l'attention des chercheurs et décideurs politiques et laissé dans l'ombre l'existence de femmes migrantes qualifiées investissant d'autres secteurs d'activité plus valorisants (études, entrepreneuriat, activités associatives, etc.). Certains auteurs (Iredale, 2001; Kofman et Raghuram, 2006) ont souligné à quel point les femmes sont absentes des études sur la migration de main-d'œuvre qualifiée.

\section{Femmes migrantes qualifiées et insertion professionnelle}

Au cours de la dernière décennie, on a vu apparaître des travaux traitant des difficultés d'insertion professionnelle des migrantes qualifiées. Les termes de "mobilité professionnelle descendante " (Creese et Wiebe, 2009), de "sous-emploi " (Asanin Dean et Wilson, 2009) et de "déqualification" (Man, 2004) se retrouvent dans la littérature pour désigner la perte de capital social et culturel associée à la difficulté d'accéder à un emploi correspondant à ses qualifications. Le terme de déqualification désigne un phénomène qui dénote le décalage

\footnotetext{
${ }^{1}$ Selon les données de la Division de la population des Nations unies pour 2010, les femmes représentaient $49 \%$ des plus de 214 millions de migrants internationaux dans le monde. Leur nombre dépassait même celui des hommes dans les pays développés, où elles constituaient $51,5 \%$ de la population migratoire internationale, contre 45,6 \% dans les pays en voie de développement (Geneste, 2013).
} 
entre d'une part le niveau de scolarité d'une personne et ses expériences professionnelles et, d'autre part, la position qu'elle occupe sur le marché de l'emploi, entraînant son déclassement social et son maintien dans une situation de dépendance (Chicha et Daraedt, 2009). Ce phénomène se traduit par des pertes de revenus et des conditions d'emploi précaires pour les migrantes, ainsi que par une moindre efficacité productive pour les entreprises. Par ailleurs, il a été montré que la déqualification œuvre à l'encontre de l'intégration sociale et favorise l'isolement (Devarennes-Megas, 2003). Nous utilisons ici la notion de déqualification comme le chômage ou l'occupation durable d'emplois inadaptés aux qualifications les plus élevées de la personne. S'y rattachent les notions de précarité financière, d’emploi temporaire ou de contrat à durée déterminée entraînant une dépendance ou une incertitude quant à l'avenir professionnel, et empêchant de faire des projets d'avenir.

Certains travaux montrent que le phénomène de la déqualification touche les femmes en général, et les migrantes en particulier (Bühler, 2010; Organisation de Coopération et de Développement économiques [OCDE], 2012). En outre, des études menées au Canada, en Allemagne et en Grande-Bretagne montrent que les femmes migrantes sont plus désavantagées que les hommes migrants face au marché du travail et sont particulièrement affectées par ce phénomène (Kofman, 2000; Rojas-Viger, 2006; Sloane, 2007).

Ce désavantage est particulièrement prononcé pour les migrantes nées dans un pays hors OCDE ou en voie de développement (Dumont et Liebig, 2005). Dans la plupart des pays, le cumul du genre et de l'origine nationale entraîne un double désavantage pour les migrantes qualifiées, qui se traduit par une déqualification plus prononcée que celle subie par leurs homologues masculins (OCDE, 2012). La lutte contre cette inégalité constitue un sujet de préoccupation croissante des gouvernements ainsi que des organisations nationales et internationales qui défendent les droits des migrants (Moreno-Fontes, 2008).

Différentes approches théoriques tentant d'expliquer le phénomène de déqualification (McGuiness, 2006), discutent de son caractère transitoire ou durable et essayent d'en identifier les causes. Plusieurs facteurs ont été mis en avant pour expliquer la déqualification des migrantes qualifiées.

a) Les facteurs structurels. L'accès au marché du travail est rendu difficile par des obstacles systémiques tels que la politique d'intégration et la nature des permis de résidence et de travail octroyés, la non-reconnaissance des diplômes et de l'expérience professionnelle acquis dans le pays d'origine, la protection par certains ordres professionnels de l'exercice de professions libérales et enfin la division sexuelle du travail confinant les femmes migrantes à des emplois peu qualifiés considérés comme féminins (Liversage, 2009).

b) Les résistances à l'embauche de la part des entreprises ou les préjugés des employeurs. Les femmes migrantes qualifiées se voient rarement confier des responsabilités de cadre ou accéder à des positions supérieures dans les entreprises, ce qui est moins le cas des hommes migrants (OCDE, 2012). Deux aspects sont à l'origine de ce désavantage, le genre et l'origine, dont le cumul est fortement préjudiciable. Différentes discriminations de genre ont été décrites, telles que le manque de flexibilité dans les horaires de travail des entreprises et la difficulté à obtenir un emploi à temps partiel qui permette de concilier vie professionnelle et vie de famille. Des stéréotypes et des préjugés culturels ou religieux jouent également un rôle discriminatoire à l'embauche puisqu'ils défavorisent un ou une candidat(e) en fonction de son origine ou disqualifient sa formation et son parcours (Fibbi, Kaya et Piguet, 2003). Une étude portant sur l'accès au marché de la formation et de l'emploi de femmes musulmanes portant le hijab à Bruxelles montre qu'elles doivent faire face à des préjugés négatifs qui les considèrent comme des personnes peu instruites et peu qualifiées (Sassi, 2013). Dans la même perspective, Vatz-Laaroussi (2008) dénonce le fait que les femmes musulmanes portant le hijab au Québec font l'objet de représentations victimisantes de la part des employeurs, étant considérées comme opprimées, soumises et victimes de la tradition. De ce fait, elles seraient perçues comme plus souples et adaptables que leurs maris, et donc davantage prêtes à accepter un emploi sous-qualifié.

c) Les facteurs familiaux. Au sein des couples où les deux conjoints menaient une carrière professionnelle avant la migration, les femmes sont souvent celles qui font des sacrifices, subissent une interruption dans leur carrière ou peinent à obtenir dans la migration une renégociation à long terme des tâches reproductives (ménage, éducation, garde des enfants). En effet, plusieurs études (Bolzman, 1996; Kofman, Kohli, Kraler et Schmoll, 2010) montrent 
que le couple trouve difficilement son équilibre hors du rapport qui lui était familier avant la migration. À cela vient s'ajouter le manque de réseau familial élargi qui, au pays, permettait de confier la garde des enfants de manière souple, informelle et gratuite à des membres de la famille et ainsi se libérer la journée. Le manque de structures d'accueil disponibles, leur coût financier élevé lorsqu'elles existent, les contraintes horaires des mères et leurs déplacements pour parvenir à jongler avec leurs diverses obligations rendent beaucoup plus compliqué pour elles l'accès au travail.

Le cumul de ces facteurs et leurs interactions constitue un frein manifeste à l'accès au monde du travail et aux possibilités d'ascension sociale des migrantes qualifiées. À cet égard, certains auteurs ont parlé de discriminations multiples ou intersectionnelles (Makkonen, 2002), concept sur lequel nous allons nous pencher à présent.

\section{Intersectionnalité et discriminations multiples}

Le concept d'intersectionnalité prend son origine dans le constat que l'individu se définit par des appartenances et des identités multiples, dans ses dimensions à la fois individuelles et collectives (Stirrat, Meyer, Ouellette et Gara, 2008). Les mouvements féministes afro-américains ont avancé l'idée que se trouver à la croisée de différents motifs de discrimination tels que le genre, la race, la classe sociale et l'orientation sexuelle produit des effets spécifiques sur les individus. Par exemple, une femme homosexuelle afro-américaine n'aura pas le même vécu qu'une homosexuelle blanche ou qu'une femme afro-américaine hétérosexuelle (Crenshaw, 1989). La combinaison de ces facteurs intrinsèquement liés et constitutifs de l'identité même de la personne produirait donc, en termes de marginalisation ou d'exclusion, un désavantage supérieur à la somme de chacun des facteurs pris isolément.

En ce qui concerne l'accès à l'emploi, Browne et Misra (2003) relèvent que lorsque le genre est croisé à d'autres caractéristiques telles que l'ethnicité ou l'origine nationale, les recruteurs combinent spontanément ces caractéristiques pour prévoir la performance des candidats à un poste. Le système de l'emploi étant déjà fortement sexué, les stéréotypes relatifs à l'origine étrangère se mêlent alors de façon étroite à ceux qui touchent plus spécifiquement les femmes.

Dans une étude réalisée à Montréal concernant les trajectoires de 44 immigrées hautement qualifiées, Chicha (2009) met en évidence que celles qui se trouvent à l'intersection de trois motifs de discrimination (genre, minorité visible et nationalité étrangère) sont plus fortement désavantagées notamment lorsqu'il s'agit d'accéder à des emplois qualifiés. Ces femmes se retrouvent enfermées dans un cercle vicieux de déqualification en raison d'obligations familiales élevées, auxquelles s'ajoutent la difficulté de faire reconnaître les diplômes étrangers, le manque de succès dans la reconversion professionnelle et la précarité des emplois occupés.

Les difficultés rencontrées par les migrantes qualifiées semblent donc se produire dans différents pays occidentaux avec des caractéristiques communes et se rapporter au phénomène de la mondialisation (Organisation internationale des migrations [OIM], 2010). Toutefois, il semble nécessaire, afin de mettre en place des mesures susceptibles d'y remédier, de considérer, au niveau local, les spécificités des politiques d'intégration des différents pays d'accueil. Dans cet article, nous allons nous intéresser plus en détail au cas de la Suisse.

\section{Immigration en Suisse et politique d'intégration}

En 2010, les étrangers représentaient $22,4 \%$ de la population résidente en Suisse, et $25 \%$ de la population active (Office fédéral de la statistique [OFS], 2010). À l'instar d'autres pays européens, la Suisse pratique actuellement une politique migratoire qui privilégie l'accueil de migrants qualifiés (Marin-Avellan et Mollard, 2012) avec le présupposé que des individus disposant d'un niveau d'éducation élevé seront plus aptes à s'intégrer. On assiste donc depuis une quinzaine d'années, à une forte progression du nombre de migrantes et migrants hautement qualifiés. De plus, le modèle dit " des cercles » privilégie les migrants issus de pays limitrophes ou de "culture occidentale " (Piguet, 2004). Ainsi, la plupart des étrangers en Suisse proviennent de pays de l'OCDE et ceux qui proviennent d'Asie, d'Amérique latine et d'Afrique représentent seulement 13,3\% d'entre eux (OFS, 2010; Bolzman, 2008). Les résultats de la récente votation populaire au niveau national, tenue le 9 février 2014 et intitulée "contre l'immigration de masse " a remis en question la libre-circulation en Suisse des travailleuses et travailleurs européens. Les partisans de cette initiative demandent que l'État fixe des plafonds pour les 
autorisations de séjour délivrées, dans le but de freiner l'immigration et de contrôler l'accès des étrangers au marché de l'emploi. Si les travailleurs européens sont touchés par cette limitation, ce sera probablement davantage le cas des personnes d'origine extra-européenne, dont le nombre était déjà contingenté avant cette votation. La situation de ces dernières sur le marché du travail deviendra encore plus compliquée.

\section{La migration des femmes en Suisse}

Actuellement, les femmes représentent $50,8 \%$ des étrangers en Suisse. Près de $43 \%$ d'entre elles sont admises pour des motifs de mariage ou de regroupement familial, contre $26 \%$ pour des raisons professionnelles (OFS, 2010). Wanner, Pecoraro et Fibbi (2005) constatent que la situation professionnelle des immigrées s'est nettement détériorée ces dernières années, comparée à celle des Suissesses (taux de chômage respectif de 9,4 \% et 3,3). Par ailleurs, l'analyse d'autres sources met en évidence des difficultés d'insertion professionnelle accrues pour les ressortissantes de pays extra-occidentaux. En effet, les migrantes originaires des pays non membres de l'OCDE affichent en moyenne un taux de chômage plus élevé que le taux global des étrangères (OCDE, 2012). Alors que le nombre de femmes provenant l'Amérique latine et de l'Afrique subsaharienne est actuellement en augmentation, les trajectoires professionnelles de ces ressortissantes extra-européennes mériteraient d'être étudiées plus en détail dans de futurs travaux de recherche.

En effet, parmi les différentes populations migrantes, la proportion de Latino-Américains installés en Suisse a doublé au cours des quinze dernières années (OFS, 2010). En particulier, on assiste à un accroissement considérable du nombre de femmes latino-américaines. Ainsi, on compte près de $65 \%$ de femmes au sein de l'immigration latino-américaine, alors que les femmes provenant d'autres pays représentent en moyenne $50 \%$ des nouvelles entrées. La surreprésentation des femmes latino-américaines, dont la majorité est âgée de 20 à 39 ans, est liée notamment à l'importance des mariages avec des ressortissants suisses, ainsi qu'à l'existence d'une forte demande de main-d'œuvre féminine dans le secteur des services (Bolzman, Carbajal et Mainardi, 2007; Riaño, 2007). Leur situation juridique est très variée : certaines vivent en Suisse sans statut légal, d'autres, venues dans le cadre du regroupement familial, pour épouser un ressortissant suisse ou pour études, ont des statuts stables (permis B, C) ou ont obtenu la nationalité suisse (OFS, 2010). Quant à leur niveau de formation, plus de la moitié des femmes latino-américaines résidant en Suisse disposent de qualifications élevées, contre $18 \%$ des autochtones (Riaño, 2003 et 2007).

Quant à la population africaine, elle représente $4 \%$ des étrangers en Suisse, ce qui constitue la proportion la plus faible de la population étrangère, bien qu'en augmentation ces dix dernières années (OFS, 2010). Ce groupe culturel est très hétérogène : presque toutes les nationalités africaines y sont représentées. La population migrante africaine reste à dominante masculine (54\% d'hommes) mais, depuis 1995, on constate une augmentation remarquable du nombre de femmes, qui a presque doublé en quinze ans. La majorité des femmes originaires d'Afrique centrale et de l'Est entrent en Suisse par la porte de l'asile, fuyant des pays en guerre et des zones de conflit armé, économiquement dévastées. Les ressortissants d'Afrique de l’Ouest migrent quant à eux surtout pour trouver de meilleures conditions de vie en Europe (Effionayi-Mäder, Pecoraro et Steiner, 2011). Les recherches sur l'insertion socioprofessionnelle des femmes migrantes africaines en Suisse sont quasi inexistantes, de même que les statistiques sur leur niveau d'études et de qualification. Cependant, une étude de Bagalwa-Mapatano (2008) sur des femmes congolaises suggère qu'elles sont particulièrement touchées par la déqualification professionnelle, mais qu'elles ont des stratégies communautaires informelles permettant la création de micro-entreprises spécialisées dans le commerce de produits africains par exemple, ce qui contribue, à terme, à leur intégration socioéconomique en Suisse. L'appartenance ethnique et communautaire semble donc ici mise à profit et tournée en atout.

\section{Insertion professionnelle et déqualification des femmes migrantes en Suisse}

L'Enquête suisse sur la population active parue en 2008 montre que la proportion d'étrangères possédant un niveau de formation tertiaire est supérieure à celui des Suissesses (22,3\% contre 15,6\%), mais cela ne se traduit pas souvent par un avantage sur le marché de l'emploi, bien au contraire. En effet, les étrangères diplômées du tertiaire ont un taux de chômage bien supérieur à celui des Suissesses, ou des hommes étrangers ou nationaux (6,1 \% contre $2,0 \%$ pour les Suissesses, 3,3\% pour les hommes étrangers et 1,4\% pour les hommes suisses). De 
manière générale, quels que soient leur situation familiale ou leur niveau d'éducation, les étrangères ont un taux d'activité inférieur aux Suissesses $(73,2 \%$ contre $78,1 \%$ ) et aux hommes, étrangers ou nationaux $(88,4 \%)$ (OFS, 2010).

Pour ce qui est du type d'emplois occupés, les étrangères sont très fortement concentrées dans des emplois subalternes et ont peu accès aux postes de cadres, alors que les hommes étrangers occupent des positions sociales proches de celles des nationaux. Par ailleurs, les emplois occupés par les étrangères semblent moins flexibles et compatibles avec une vie de famille que ceux occupés par les Suissesses : en effet, le travail à temps partiel est nettement moins répandu chez les étrangères ( $27 \%$ contre $44 \%)$, alors que le travail à plein temps s'observe aussi souvent chez les étrangères que chez les Suissesses (32 \% contre $31 \%$ ) (OCDE, 2012).

Ce désavantage lié à l'origine vient s'ajouter à celui lié à la condition féminine. En effet, de manière générale, les femmes en Suisse sont moins bien intégrées dans le monde du travail que les hommes. Plusieurs indicateurs statistiques reflètent cette inégalité sociale : des salaires plus bas, une sous-représentation des femmes dans les instances dirigeantes, parmi les titulaires de doctorats ou encore parmi les responsables du système scientifique (Bühler, 2010).

$\mathrm{Au}$ vu de ces données, il semble que les migrantes sont confrontées en Suisse à un double handicap dans leurs tentatives d'accéder au marché de l'emploi, en raison de leur origine et de leur sexe. Ce désavantage se traduit à la fois par le chômage et par la déqualification. Celle-ci affecte plus particulièrement les primo-arrivantes nées à l'étranger et ayant émigré après l'âge de 15 ans (Pecoraro, 2005), tout comme les femmes nées dans un pays hors OCDE (Dumont et Liebig, 2005).

Ces constats ont conduit plusieurs chercheurs à analyser les types de déqualification en Suisse. Wirz et Atuken (2005) en distinguent deux: le premier concerne les individus travaillant dans leur domaine d'études mais à un niveau inférieur, alors que le second correspond aux personnes travaillant dans un autre champ que celui de leur formation initiale. Le premier type pourrait s'expliquer par des rigidités systémiques du marché du travail (manque d'information, contrainte à la mobilité professionnelle, discriminations liées au genre ou à l'origine). Le second type de déqualification serait dû à l'incapacité pour le demandeur d'emploi de mettre en avant certaines caractéristiques personnelles en lien avec le poste convoité (motivation, leadership, diplômes, expérience, savoirfaire, etc.). Les auteurs observent que la déqualification touche en particulier les mères d'enfants en bas âge par manque de soutien en matière de garde d'enfants. Mais ils considèrent qu'il s'agit d'un problème passager et refusent de parler de discrimination touchant spécifiquement les femmes ou les migrants.

Contrairement à ce point de vue, deux études empiriques récentes soutiennent que la déqualification est une réalité durable que doivent affronter les migrantes qui cherchent à intégrer le marché de l'emploi en Suisse. L'étude de Riaño et Baghdadi (2007) a porté sur 57 femmes qualifiées, âgées en moyenne de 40 ans, nées à l'étranger et résidant en Suisse depuis 11 ans en moyenne, originaires d'Amérique latine, du Moyen-Orient et d'Europe de l'Est et vivant dans trois cantons alémaniques. Leurs motifs d'immigration étaient essentiellement le mariage, le regroupement familial et l'asile. Cette enquête montre qu'en dépit de leur niveau de qualification élevé, 82 \% des femmes interrogées ne sont pas parvenues à faire valoir leur capital socioculturel afin d'obtenir un poste stable à responsabilités. Celles qui occupaient un poste en accord avec leur niveau de compétences avaient, pour la plupart, des contrats à durée déterminée. Les auteurs insistent sur l'importance du lieu d'obtention des diplômes, ceux obtenus à l'étranger semblant plus difficiles à valoriser.

L'étude de Chicha et Deraedt (2009), conduite à Genève, porte sur 23 migrantes qualifiées, nées à l'étranger, résidant en Suisse depuis au moins 5 ans, d'origines géographiques différentes. Les objectifs de l'étude étaient d'identifier les stratégies mises en place pour faire reconnaître les diplômes, puis détecter la présence de déqualification et retracer les trajectoires professionnelles (ascendante, stagnante ou menant au chômage). Toutes les participantes avaient obtenu leurs diplômes à l'étranger et avaient occupé un emploi en Suisse, même de courte durée. Trois degrés de déqualification ont été retenus : les migrantes occupant un emploi en accord avec leurs qualifications, les migrantes détenant un emploi pour lequel elles étaient surqualifiées et celles détenant un emploi qui ne requiert aucun diplôme ou qualification. La concordance entre qualification et emploi est observée 
dans seulement $30 \%$ des cas. Les auteurs concluent que la déqualification est un phénomène qui s'installe de manière durable, persistant parfois même après 10 ans de séjour en Suisse.

Dans les deux études mentionnées, les auteurs attribuent la déqualification à la fois à des rigidités institutionnelles (permis de séjour restrictifs, accès limité aux postes dans la fonction publique) et à diverses formes de discriminations. Ces rigidités sont ancrées aussi bien dans les législations que dans les pratiques institutionnelles et les représentations sociales, ce qui explique leur caractère durable. À cela s'ajoute un facteur relatif à la familiarité avec la culture du pays d'accueil, appelé littératie (literacy): les femmes sont parfois désavantagées par leur méconnaissance de la langue ou de la région d'accueil, ce qui augmente la probabilité de leur déqualification (Bolzman, 1996). Par ailleurs, la succession de deux ou trois emplois peu qualifiés diminue les chances d'ascension ultérieure.

Une troisième étude plus récente effectuée à Genève par Marin-Avellan et Mollard (2012) se centre sur l'impact de la déqualification, actuelle ou passée, sur le bien-être psychologique et social des femmes concernées. Elle a porté sur 35 migrantes, originaires du "Sud », habitant à Genève et disposant d'un permis de séjour valable. Les résultats montrent que, depuis leur arrivée à Genève, $40 \%$ des participantes avaient traversé des périodes de tension et de détresse psychologique qu'elles attribuaient à diverses situations en lien avec la recherche d'emploi infructueuse et les relations difficiles avec l'office du chômage. Plus le degré de la déqualification était important, plus la détresse psychologique rapportée était intense, ainsi que le sentiment de discrimination. Toutefois, cette étude présente un certain nombre de faiblesses méthodologiques, en particulier la grande hétérogénéité des caractéristiques des participantes. La durée de séjour en Suisse était très variable, allant de 2 à 25 ans. Cela rend d'autant plus problématique le fait que l'état de santé mentale est évalué de manière rétrospective. En outre, certaines participantes incluses dans l'étude n'avaient pas une bonne maîtrise du français, ce qui est un élément qui les défavorise objectivement lors de la recherche d'un emploi.

En résumé, les travaux existants en Suisse comportent plusieurs écueils méthodologiques. Leurs résultats sont issus d'échantillons très hétérogènes, qui comptent des femmes à des moments très différents de leur parcours migratoire, à des niveaux différents de maîtrise de la langue locale et avec des statuts légaux différents, conditions déterminantes dans la recherche d'un emploi. Ceci rend difficile l'interprétation des résultats du fait du nombre important de variables non contrôlées. Afin de compléter nos connaissances et d'améliorer les réponses à ce problème, il serait souhaitable d'interroger des femmes ayant des caractéristiques sociodémographiques plus homogènes et bénéficiant d'autorisations de séjour stables et de travail. II paraît en outre important de réduire les biais de sélection en recrutant les sujets dans des milieux variés.

Les données issues du champ de la sociologie du travail investiguent peu les facteurs qui influencent l'impact qu'ont les obstacles et les difficultés d'insertion professionnelle sur la santé mentale des femmes migrantes. De même, dans le champ de la psychologie clinique, peu de travaux ont tenté de mieux cerner le rôle de facteurs contextuels (comme l'insatisfaction professionnelle) parmi les conditions qui influencent négativement la santé mentale des migrants. Dans ce qui suit, nous allons nous appuyer sur une vaste littérature internationale mettant en relation emploi, discrimination perçue, qualité de vie ou satisfaction et santé mentale, dans une perspective qui tente de mieux articuler les facteurs individuels et sociaux susceptibles de favoriser la détresse psychologique qui peut résulter de la déqualification ou, au contraire, de préserver de cette détresse.

\section{L'impact de la déqualification sur la santé mentale des femmes migrantes}

La grande majorité des travaux sur la santé mentale des migrants s'intéresse à l'impact des facteurs psychosociaux affectant les personnes en situation de grande précarité, tandis que les migrants appartenant à des milieux moins défavorisés sont largement négligés dans la littérature (Chicha, 2009). Il est dorénavant reconnu que si à leur arrivée les migrants présentent un meilleur état de santé que la population générale (phénomène désigné sous le nom de " healthy migrant effect »), celui-ci se détériore parfois au fil des années (McDonald et Kennedy, 2004). Par ailleurs, on observe d'importantes variations selon les origines nationales (Gabadinho, Wanner et Dahinden, 2007). Un courant de recherche actuel explique le taux important de morbidité présenté par les migrants plusieurs années 
après leur arrivée et les variations inter-groupes par l'impact psychologique des expériences de discrimination et de racisme vécues parfois par les membres de ces groupes (Hyman, 2009; Pittman, 2011).

D'autre part, un courant de recherche en psychologie interculturelle s'intéresse au lien entre santé mentale des migrants et processus d'acculturation. En effet, d'un point de vue psychologique, la migration entraîne une perte de repères internes et une déstabilisation des routines socioculturelles. Le processus d'acculturation décrit par Berry (1980) permet une progressive maîtrise des codes, normes et valeurs de la société d'accueil, de façon à être accepté dans ce groupe et à y évoluer avec aisance en tant que membre à part entière. Vue sous cet angle, l'acculturation est le processus qui mène à l'adaptation (Sabatier et Berry, 1994). Soulignons toutefois que les stratégies d'acculturation décrites par Berry (1980) ne relèvent pas uniquement du ressort individuel mais doivent composer avec la réalité sociopolitique, les attitudes des résidants et les difficultés rencontrées lors de l'insertion dans la société d'accueil (Bourhis et Leyens, 1994). Ainsi, le processus d'acculturation comporte une dimension transactionnelle et liée au milieu de vie, ce qui en fait une variable médiatrice entre les caractéristiques personnelles du migrant, les facteurs environnementaux et l'état de santé mentale de l'individu à l'issue de la migration (Cuellar, 2000; Goguikian Ratcliff, 2009).

La difficulté à trouver un emploi, la déqualification, l'isolement social ainsi que les écarts importants entre les attentes professionnelles pré-migratoires et la réalité rencontrée sur le marché du travail à l'arrivée sont susceptibles de produire des sentiments de découragement, d'injustice, d'autodépréciation, de dévalorisation socioprofessionnelle et un manque de satisfaction par rapport à l'existence actuelle (Cardu, 2008 et 2012) et, à terme, ils sont donc susceptibles de nuire à la santé mentale (Devarenne-Megas, 2003).

En effet, dans un rapport mandaté par l'OMS, se basant sur plusieurs études européennes et américaines, Wilkinson et Marmot (2004) rappellent qu'il est préjudiciable pour la santé d'un individu de ne pas pouvoir utiliser ses compétences et de ne pouvoir disposer que d'un pouvoir limité de décision. Le risque est accru pour les postes où la charge de travail est importante et le niveau d'autonomie faible. Un emploi précaire ou une forte insatisfaction professionnelle peuvent être aussi néfastes que le chômage et sont associés à des taux élevés de dépression et d'anxiété (Burchell, 1994) et ils contribuent à un état de stress chronique, d'autant plus dommageable qu'il se prolonge. À l'inverse, une revue récente de la littérature par Lennon et Limonic (2010) souligne l'effet protecteur d'un emploi adéquat et de conditions de travail satisfaisantes sur le sentiment de bienêtre et sur la santé mentale, en tant que composantes de la qualité de vie.

Ainsi, la déqualification des femmes migrantes peut engendrer une certaine précarité matérielle et sociale et par là même affecter négativement leur qualité de vie. Des études longitudinales prospectives ont montré que la qualité de vie perçue permet de prédire l'apparition de symptômes somatiques ou de troubles mentaux (Schnurr, Hayes, Lunney, McFall et Uddo, 2006). Par ailleurs, Wang et ses collaborateurs (2010) soulignent que, chez les migrants, la qualité de vie est sous-tendue par les objectifs personnels, par la perception du statut social ainsi que par le sentiment de discrimination.

\section{Le rôle du sentiment de discrimination}

La discrimination perçue est le sentiment subjectif d'être traité de manière injuste ou inéquitable par rapport à d'autres dans la vie quotidienne, à cause de son appartenance à une minorité. Ce concept fait référence à la mesure dans laquelle les personnes perçoivent ou jugent certains comportements émis envers eux-mêmes ou leur groupe d'appartenance comme étant de la discrimination, et ce, indépendamment du fait que cette perception des choses reflète ou non la réalité objective (Taylor, Wright et Porter, 1994). De ce point de vue, la perception de la discrimination est directement liée à l'appartenance des personnes à un groupe social ou culturel minoritaire faisant habituellement l’objet de préjugés négatifs dans une société donnée.

Cette perception est donc étroitement liée aux différences de statut social et porte atteinte à l'estime de soi et, de manière plus générale, à la santé physique et mentale (Major, O’Brien, Kaiser et McCoy, 2007). Les expériences de discrimination peuvent représenter des stress quotidiens, agissant de manière durable, stress auxquels les personnes sont exposées dans différentes sphères: emploi, avancement professionnel, atmosphère au travail, 
logement, interdiction d'entrée dans des lieux publics, difficultés à obtenir un emprunt financier, mauvais traitement de la part des fonctionnaires d'institutions publiques, etc.

Un nombre croissant d'études effectuées sur des minorités ethniques résidant dans différents pays montre qu'il existe une relation entre un sentiment de discrimination et différents indicateurs de mauvaise santé physique (Williams, Neighbors et Jackson, 2003) et mentale (Karlsen et Nazroo, 2002). À Montréal, par exemple, l'enquête Santé Québec sur les communautés culturelles a mis en évidence une association significative entre la perception de la discrimination et la présence de détresse psychologique (Rousseau et Drapeau, 2004).

Le lien entre discrimination perçue et santé mentale a également été étudié en comparant l'intensité de ce sentiment chez différents groupes ethniques. Deux études comparatives réalisées en Finlande (Liebkind et Jasinskaja-Lahti, 2000; Jasinskaja-Lahti, Liebkind, Jaakola et Reuter, 2006) montrent qu'indépendamment de la durée du séjour dans le pays d'accueil, la discrimination perçue est plus élevée chez les personnes appartenant à une minorité visible et considérées comme plus distantes du point de vue culturel.

Il est bien établi que la présence de réseaux de soutien social et communautaire permet de faire face à ces expériences de discrimination et de se protéger contre le stress (Noh et Kaspar, 2003), faute de quoi le sentiment de discrimination est exacerbé. L'utilité des contacts sociaux comme facteurs de protection contre le stress est accrue, qu'ils s'effectuent avec des autochtones ou des compatriotes (Jasinskaja-Lahti, Llebkind, Jaakola et Reuter, 2006).

\section{Insatisfaction professionnelle : décalage entre attentes professionnelles pré-migratoires et réalité post-} migratoire

Les attentes pré-migratoires ont été étudiées comme un prédicteur de la qualité de vie après la migration. Cherchant à identifier les sources du stress d'acculturation chez les migrants, Williams et Berry (1991) proposent le concept de "goal-striving stress", que l'on pourrait traduire par stress découlant d'un but entravé ou d'une aspiration frustrée. Un lien entre niveau élevé d'aspirations prémigratoires et intensité des symptômes psychologiques après la migration a pu être établi dans des études américaines sur de jeunes immigrés vietnamiens (McKelvey et Webb, 1996). Toutefois, ce lien reste controversé puisque certaines études montrent qu'un optimisme de départ et des attentes élevées agissent positivement sur la motivation à s'intégrer et sont des facteurs protecteurs de la psychopathologie, alors que d'autres travaux (Williams et Berry, 1991) indiquent, à l'inverse, que des attentes élevées et irréalistes augmentent le risque de détresse psychique une fois dans le pays d'accueil.

Le décalage entre les attentes de départ et la réalité à l'arrivée est fortement tributaire des conditions officielles qui régissent l'accès au monde du travail dans le pays d'accueil, autrement dit à sa politique migratoire, plus ou moins favorable (ou défavorable) aux nouveaux arrivants. Les impératifs économiques et les difficultés rencontrées pour obtenir un emploi correspondant à leur niveau de qualification incitent les migrants à accepter des emplois pour lesquels ils sont surqualifiés. La discordance entre les objectifs que l'on s'était fixés avant la migration et ce que l'on est réellement en mesure d'accomplir après-coup dans le pays d'accueil peut générer stress, insatisfaction et souffrance psychologique (Nazroo, McKenzie, Bhui et Weich, 2005). C'est la raison pour laquelle Zhang, Li, Fang et Xiong (2009) proposent de considérer non pas les attentes pré-migratoires en tant que telles, mais le décalage entre attentes pré-migratoires et réalité post-migratoire. II s'agit là d'une variable individuelle de type cognitif, puisqu'elle découle d'une activité de jugement, et qui comporte une dimension subjective au même titre que le sentiment de discrimination.

\section{Stratégies de résilience}

Face aux obstacles et à la réalité socioprofessionnelle locale, les femmes migrantes, au Québec, développent des stratégies spécifiques de résistance et de résilience qui guident leurs choix relatifs à l'insertion : accepter un emploi déqualifié, reprendre des études, privilégier les liens avec la communauté nationale ou familiale, renoncer à travailler et rester au foyer, etc. (Vaatz-Laroussi, 2008; Cardu, 2012). II est indéniable que leurs choix tiennent compte de la situation professionnelle du conjoint et de l'âge des enfants. À cet égard, Vaatz-Laroussi (2008) fait 
I'hypothèse que les femmes mettent en œuvre des stratégies à plus long terme qui seraient ancrées dans des attitudes et des valeurs positives (optimisme, respect, engagement). Dans un contexte différent, en Belgique, les stratégies sont similaires: les femmes opposent trois stratégies principales face à la discrimination et aux obstacles : la renonciation à leurs aspirations, le contournement de la difficulté (en se retirant du marché du travail « classique » pour s'orienter vers des secteurs ou des statuts qui paraissent plus accessibles) et la résistance (en refusant les pressions de la société par des actions telles que refuser un emploi jugé non compatible avec ses valeurs, persévérer, négocier, persuader, développer son capital social) (Sassi, 2013).

\section{Conclusions et perspectives}

Il ressort de la revue de la littérature que la déqualification des femmes migrantes est un processus dont les effets sont à la fois psychologiques et sociaux. Il serait intéressant de saisir la dynamique de ce processus, les conditions de son installation et les stratégies spécifiques mises en place pour y faire face par différents groupes de femmes migrantes en Suisse. Les questions suivantes restent à approfondir :

- Le phénomène de déqualification touche-t-il plus certaines catégories de migrantes que d'autres ?

- Quel est l’impact psychologique de la déqualification?

- Existe-t-il des parcours types qui conduisent les femmes migrantes à la déqualification, ou des délais au-delà desquels l'accès à un emploi correspondant à leurs qualifications devient de moins en moins possible?

- Quelles sont les stratégies qui s'avèrent payantes chez les femmes migrantes qui parviennent à échapper à la déqualification ou à en réduire la durée?

En outre, le rôle modérateur joué par certains facteurs spécifiques aux migrants et migrantes (acculturation, attentes pré-migratoires, discrimination perçue) est encore peu étudié. L'identification des facteurs de risque individuels et environnementaux et leurs interactions, l'étude des trajectoires pouvant mener à une déqualification plus ou moins durable ainsi que la comparaison entre différents groupes de femmes migrantes et un groupe de femmes non migrantes permettrait d'envisager des programmes d'accompagnement (associatifs, institutionnels) plus ciblés et efficaces afin de prévenir la déqualification de femmes qualifiées et ses possibles effets délétères en matière de santé mentale.

\section{Bibliographie}

Asanin Dean, J. et Wilson, K. (2009). «Education? It is irrelevant to my job now. It makes me very depressed...» : exploring the health impacts of under/unemployment among highly skilled recent immigrants in Canada. Ethnicity and Health, 14(2), 185-204.

Bagalwa-Mapatano, J. (2008). Place et rôle de la femme dans la diaspora congolaise de Suisse 1980-2000. L'Africain, 233, 20-30.

Berry, J.-W. (1980). Social and cultural Change. Dans H. Trandis et R. Brislin (dir.), Handbook of Cross-Cultural Psychology, vol. 5 (p. 211-279). Boston : Allyn and Bacon.

Bolzman, C. (1996). Sociologie de l'exil : une approche dynamique. L'exemple des réfugiés chiliens en Suisse. Zurich : Seismo.

Bolzman, C. (1997). Aux frontières du public et du privé : la négociation des rôles familiaux en situation d'exil. Dans P. Béday et C. Bolzman (dir.), On est né quelque part mais on peut vivre ailleurs. Familles, migrations, cultures, travail social (p. 79-100). Genève : IES Institut d'études sociales.

Bolzman, C. (2008). Travailleurs étrangers sur le marché du travail Suisse : quels modes d'incorporation ?. Journal of International Migration and Integration, 8(2), 357-373.

Bolzman, C., Carbajal, M. et Mainardi, G. (dir.) (2007). La Suisse au rythme latino. Genève : Institut d'études sociales (IES).

Bourhis, R. et Leyens, J.-P. (1994). Stéréotypes, discrimination et relations entre groupes. Liège : Mardaga. 
Browne, I. et Misra, J. (2003). The intersection of gender and race in the labor market. Annual Review of Sociology, 29, 487-513.

Bühler, E. (2010). Atlas suisse des femmes et de l'égalité. Zurich : Seismo.

Burchell, B. (1994). The effects of labour market position, job insecurity, and unemployment on psychological health. Dans D. Gallie, C. Marsh et C. Vogler (dir.), Social change and the experience of unemployment (p. 188-212). Oxford: Oxford University Press.

Cardu, H. (2008). Femmes immigrantes, soutien et situation d'insertion socioprofessionnelle... entre ruptures et continuités. Dans M. Vatz-Laaroussi, C. Bolzman, et M. Lahlou (dir.), Familles migrantes au gré des ruptures. Tisser la transmission (p. 293-302). Lyon : L’interdisciplinaire.

Cardu, H. (2012). Resilience strategies used by immigrant women facing professional deskilling in Quebec. Dans International Organization for Migration (dir.), Crushed hopes: underemployement and deskilling among skilled migrant women (p. 137-161). Genève : Organisation internationale pour les migrations (IOM).

Catarino, C. et Morokvasic, M. (2005). Femmes, genre, migration et mobilités. Revue européenne des migrations internationales, 21(1), 7-27.

Chaïb, S. (2001). Facteurs d'insertion et d'exclusion des femmes immigrantes dans le marché du travail en France : quel état des connaissances ? Document de travail élaboré pour la Confédération française démocratique du travail.

Chicha, M. (2009). Le mirage de l'égalité : les immigrées hautement qualifiées à Montréal [en ligne]. http://archives.enap.ca/bibliotheques/2009/11/30118647.pdf

Chicha, M. et Deraedt, E. (2009). Genre, migration et déqualification : des trajectoires contrastées. Étude de cas de travailleuses migrantes à Genève. Dans Cahiers des migrations internationales 97. Genève : Organisation internationale du travail.

Creese, G. et Wiebe, B. (2009). 'Survival employment': Gender and deskilling among African immigrants in Canada. Oxford: Blackwell Publishing.

Crenshaw, K. (1989). Demarginalizing the Intersection of Race and Sex. A Black Feminist Critique of Antidiscrimination Doctrine, Feminist Theory, and Antiracist Politics. University of Chicago Legal Forum, p. 139-167.

Cuellar, I. (2000). Acculturation and mental health : ecological transactional relations of adjustment. Dans I. Cuellar et F. Paniagua (dir.), Handbook of multicultural mental health: Assessment and treatment of diverse populations, (p. 45-62). San Diego : Academic Press.

Devarenne-Megas, H. (2003). Psychopathologie et insertion sociale des migrants polonais en France. Revue européenne des migrations internationales, 19(1), 2-20.

Dumont, J. et Liebig, T. (2005). Labour market integration of immigrant women: overview and recent trends. The $O E C D$ and European Commission Seminar on migrant women and the labour market: Diversity and challenges. Bruxelles : OCDE Publishing.

Effionayi-Mäder, D., Pecoraro, M. et Steiner, I. (2011). La population subsaharienne en Suisse : un aperçu démographique et socioprofessionnel. Neuchâtel : Forum suisse des migrations.

Fibbi, R., Kaya, B. et Piguet, E. (2003). Le passeport ou le diplôme? Etude des discriminations à l'embauche des jeunes issus de la migration. Neuchâtel : Forum suisse des migrations.

Gabadinho, A., Wanner, P. et Dahinden, J. (2007). La santé des populations migrantes en Suisse : une analyse des données du GMM. Neuchâtel : Swiss forum for migration and population studies (SFM).

Geneste, A. (2013, 8 mai). Le nouveau visage féminin de la migration [en ligne]. Le Monde. http://www.lemonde.fr/ameriques/article/2013/05/08/le-nouveau-visage-feminin-de-lamigration_3173506_3222.html 
Goguikian Ratcliff, B. (2009). Conclusion. Pour une clinique plus sociale du traumatisme et de l'exil. Dans B. Goguikian Ratcliff et O. Strasser (dir.), Clinique de l'exil : chroniques d'une pratique engagée (p. 185-196). Genève : Georg.

Hyman, M. (2009). Responsible ads: A workable ideal. Journal of Business Ethics, 87(2), 199-210.

Iredale, R. (2001). The migration of professionals: theories and typologies. International Migration, 39(5), 7-24.

Jasinskaja-Lahti, I., Liebkind, K., Jaakola, M. et Reuter, M. (2006). Perceived discrimination, social support networks, and psychological well-being among three immigrant groups. Journal of Cross-Cultural Psychology, 73(3), 293-311.

Karlsen, S. et Nazroo, J. (2002). Relation between racial discrimination, social class, and health among ethnic minority groups. Americal Journal of Public Health, 92(4), 624-631.

Kofman, E. (2000). Gender and international migration in Europe. New York, NY : Routledge.

Kofman, E. (2004). Genre et migration internationale : critique du réductionnisme théorique. Dans C. Zaidman et P. Bachelet (dir.), Genre, travail et migrations en Europe (p. 81-97). Paris : Cahiers du CEDREF.

Kofman, E. (2009). Gendered migrations and the globalization of social reproduction and care: new dialogues and directions. Dans E. Schrouver et E. Yeo (dir.), Gender and migration in global, historical and theoretical perspective (p. 118-139). Taylor and Francis (Routledge).

Kofman, E., Kohli, M., Kraler, A. et Schmoll, C. (2010). Gender, Generations and the Family in International Migration. Amsterdam : Amsterdam University Press.

Kofman E. et Raghuram, P. (2006). Gender and global labour migrations : Incorporating skilled workers. Antipode, 38(2), 282-303.

Lennon, M. et Limonic, L. (2010). Work and Unemployment as stressors. Dans T. Scheid et T. Brown (dir.), $A$ handbook for the study of mental health: Social contexts, theories and systems (p. 213-225). Cambridge : Cambridge University Press.

Liebkind, K. et Jasinskaja-Lahti, I. (2000). The influence of experiences of discrimination on psychological stress: a comparison of seven immigrant groups. Journal of Community and Applied Social Psychology, 10, 1-16.

Liversage, A. (2009). Vital conjunctures, shifting horizons : high-skilled female immigrants looking for work. Work Employment Society, 120-141.

Major, B., O’Brien, L., Kaiser, C. et McCoy, K. (2007). Perceived discrimination as a worldview threat or worldview confirmation: implications for self-esteem. Journal of Personality and Social Psychology, 92(6), 1068-1086.

Makkonen, T. (2002). Multiple, compound and intersectional discrimination: bringing the experiences of the most marginalized to the fore. Institute for Human Rights, Åbo Akademi University.

Man, G. (2004). Gender, work and migration: Deskilling Chinese immigrant women in Canada. Women's Studies International Forum, 27(2), 135-148.

Marin-Avellan, L. et Mollard, B. (2012). L'impact psychosocial du sous-emploi sur la vie des femmes migrantes qualifiées travaillant à Genève (Suisse). Genève : Organisation internationale pour les migrations.

McDonald, J. et Kennedy, S. (2004). Insights into the 'healthy immigrant effect' : health status and health service use of immigrants to Canada. Social Science and Medicine, 59, 1613-1627.

McGuiness, S. (2006). Overeducation in the labour market. Journal of Economic Survey, 20(3), 292-299.

McKelvey, R. et Webb, J. (1996). Pre-migratory expectancies and post-migratory mental health symptoms in Vietnamese Amerasian youth. Journal of the American Academy of Child and Adolescent Psychiatry, 35(2), 240-245.

Moreno-Fontes, G. (2008). Female migrant workers' situation in the labour market [en ligne]. http://www.immigrantwomen.gr/portal/images/arthra/labour.pdf

Alterstice-Revue Internationale de la Recherche Interculturelle, vol. 4, $n^{\circ} 2$ 
Morokvasic, M. (2003). Transnational mobility and gender: a view from post-wall Europe. Dans M. Morokvasic, U. Erel et K. Shinozaki (dir.), Crossing borders and shifting boundaries: Gender on the Move, vol. 1 (p. 101 133). Opladen : Leske and Budrich.

Nazroo, J., McKenzie, K., Bhui, K. et Weich, S. (2005). Racism, psychosis and common mental disorder among ethnic minority groups in England. Psychological Medicine, 35(12), 1795-1893.

Noh, S. et Kaspar, V. (2003). Perceived discrimination and depression: Moderating effects of coping, acculturation, and ethnic support. American Journal of Public Health, 93, 232-238.

Office fédéral de la statistique (OFS) (2010). La population étrangère en suisse. Neuchâtel : OFS.

Organisation de coopération et de développement économiques (OCDE) (2012). Perspectives des migrations internationales [en ligne]. http://dx.doi.org/10.1787/migr_outlook-2012-fr

Organisation internationale des migrations (OIM) (2010). État de la migration dans le monde 2010. L'avenir des migrations : renforcer les capacités face aux changements. Genève : OIM.

Pecoraro, M. (2005). Les migrants hautement qualifiés en Suisse. Migrants et marché du travail en Suisse, 6, 71108.

Piguet, E. (2004). L'immigration en Suisse : 50 ans d'entreouverture. Lausanne : Presses Polytechniques et Universitaires romandes.

Pittman, C. (2011). Getting mad but ending sad: the mental health consequences for African Americans using anger to cope with racism, Journal of Black Studies, 42, 1106-1124.

Riaño, Y. (2003). Migration of skilled Latin American women to Switzerland and their struggle for integration. Dans Y. Mutsuo (dir.), Latin American emigration : Interregional comparison among North America, Europe and Japan. JCAS Symposium Series 19. Osaka : Japan Centre for Area Studies.

Riaño, Y. (2007). Migration de femmes latino-américaines universitaires en Suisse. Dans C. Bolzman, M. Carbajal et G. Mainardi (dir.), La Suisse au rythme latino (p. 115-136). Genève : IES.

Riaño, Y. et Baghdadi, N. (2007). Understanding the labour market participation of skilled immigrant women in Switzerland: the role of class, ethnicity and gender. Journal of International Migration and Integration, 8, 163-183.

Rojas-Viger, C. (2006). Femmes professionnelles latino-américaines à Montréal : conditions d'insertion dans le milieu universitaire et au marché du travail. Les Cahiers du Gres, 6(1), 25-43.

Rousseau, C. et Drapeau, A. (2004). Premigration exposure to political violence among independent immigrants and its association with emotional distress. Journal of Nervous and Mental Disease, 192(12), 852-856.

Sabatier, C. et Berry, J. (1994). Immigration et acculturation. Dans R. Bourhis et J.-P. Leyens (dir.), Stéréotypes, discrimination et relations intergroupes (p. 261-291). Liège : Mardaga.

Sassi, K. (2013). Stratégies mises en place par les femmes voilées universitaires pour accéder et se maintenir en emploi. Dans A. Manço et C. Barras (dir.), La diversité culturelle dans les PME. Accès au travail et valorisation des ressources (p. 97-110). Paris : L'Harmattan.

Schnurr, P., Hayes, A., Lunney, C., McFall, M. et Uddo, M. (2006). Longitudinal analysis of the relationship between symptoms and quality of life in veterans treated for posttraumatic stress disorder. Journal of Consulting and Clinical Psychology, 74, 707-713.

Sloane, P. (2007). Overeducation in the United Kingdom. Policy forum: education and skill mismatches in the labour market. The Australian Economic Review, 40(3), 286-291.

Stirratt, M., Meyer, I., Ouellette, S. et Gara, M. (2008). Measuring identity multiplicity and intersectionality: Hierarchical classes analysis (HICLAS) of sexual, racial, and gender identities. Self and Identity, 7(1), 89-111. 
Taylor, D., Wright, S. et Porter, L. (1994). Dimension of perceived discrimination: The personal/group discrimination discrepancy. Dans M. Zanna et J. Olson, The Psychology of Prejudice: The Ontario Symposium (p. 233-255). Hillsdale, ND : Erlbaum.

Vatz-Laaroussi, M. (2008). Du Maghreb au Québec : accomodements et stratégies. Revue internationale Travail, genre et société, 20(novembre), 47-66.

Wang, J., Cui, Z.-T., Cui, H.-W., Wei C.-N., Harada, K., Minamoto, K. et Ueda, A. (2010). Quality of life associated with perceived stigma and discrimination among the floating population in Shanghai, China: qualitative study. Health Promotion International, 25(4), 394-402.

Wanner, P., Pecoraro, M. et Fibbi, R. (2005). Femmes étrangères et marché du travail. Dans W. Haug et P. Wanner (dir.), Migrants et marché du travail (p. 17-70). Neuchâtel : Office Fédéral de la Statistique.

Wilkinson, R. et Marmot, M. (2004) (dir.). Les déterminants sociaux de la santé. Les faits ( $2^{\mathrm{e}}$ éd.). Copenhague : Bureau régional de l'OMS pour l'Europe.

Williams, C. et Berry, J. (1991). Primary prevention of acculturative stress among refugees. The American Psychologist, 46, 632-641.

Williams, D., Neighbors, H. et Jackson, J. (2003). Racial/ethnic discrimination and health: findings from community studies. American Journal of Public Health, 93, 200-208.

Wirz, L. et Atuken, E. (2005). An analysis of perceived overqualification in the Swiss labor market. Economics Bulletin, 9(2), 1-10.

World Health Organization Quality of Life Group (WHOQOL) (1995). The World Health Organization Quality of Life Assessment Instrument (WHOQOL): position paper from the World Health Organization. Social science and medicine, 41(10), 1403-1409.

Zhang, J., Li, X., Fang, X. et Xiong, Q. (2009). Discrimination experience and quality of life among rural-to-urban migrants in China: the mediation effect of expectation reality discrepancy. Quality of Life Research, 18, 291300. 